(2) Open Access Full Text Article

\title{
Dysregulation of Neuregulin- I/ErbB signaling in the hippocampus of rats after administration of doxorubicin
}

This article was published in the following Dove Press journal:

Drug Design, Development and Therapy

\author{
Dehua Liao',2 \\ Yujin Guo ${ }^{3}$ \\ Daxiong Xiang ${ }^{2}$ \\ Ruili Dang ${ }^{3}$ \\ Pengfei $X_{u^{3}}$ \\ Hualin $\mathrm{Cai}^{2}$ \\ Lizhi Cao' \\ Pei Jiang ${ }^{3}$ \\ 'Department of Pharmacy, Hunan \\ Cancer Hospital, ${ }^{2}$ Department \\ of Pharmacy, Institute of Clinical \\ Pharmacy \& Pharmacology, Second \\ Xiangya Hospital, Central South \\ University, Changsha, ${ }^{3}$ Department \\ of Pharmacy, Jining First People's \\ Hospital, Jining Medical University, \\ Jining, People's Republic of China
}

Correspondence: Pei Jiang Department of Pharmacy, Jining First People's Hospital, Jining Medical University, No. 6, Jiankang Road, Jining 272000, Shandong, People's Republic of China

Email jiangpeicsu@sina.com
Objective: Long-term use of doxorubicin (Dox) can cause neurobiological side effects associated with depression, but the underlying mechanisms remain equivocal. While recent evidence has indicated that Neuregulin-1 (NRG1) and its ErbB receptors play an essential role in neural function, much is still unknown concerning the biological link between the NRG1/ErbB pathway and the Dox-induced neurotoxicity. Therefore, we examined the protein expression of NRG1 and ErbB receptors in the hippocampus of rats following Dox treatment.

Materials and methods: The drug was administered every 2 days at a dose of $2.5 \mathrm{mg} / \mathrm{kg}$, and the animals in different groups were treated with intraperitoneal injection for three or seven times, respectively.

Results: Our data showed that the rats treated with Dox for seven times (DoxL group) exhibited depression-like behaviors, whereas the short-term treatment (DoxS group) had no effect on the behavioral changes. Dox treatment also induced the neural apoptosis with more severe neurotoxicity. Intriguingly, the expression of NRG1 and the ratio of pErbB4/ErbB4 and pErbB2/ErbB2 were significantly decreased in the DoxL group, but enhanced activation of ErbB receptors was observed in the DoxS group. In parallel, administration of Dox for seven times suppressed the downstream Akt and ERK phosphorylation, while the Akt phosphorylation was enhanced with the administration of Dox for three times.

Conclusion: Our data first showed the Dox-induced alterations of the NRG1/ErbB system in the hippocampus, indicating the potential involvement of the NRG1/ErbB pathway in the Dox-induced nervous system dysfunction.

Keywords: chemotherapy, neurotoxicity, depression, NRG1/ErbB system, Akt, ERK

\section{Introduction}

Doxorubicin (Dox) is an effective anthracycline chemotherapeutic developed for the treatment of solid tumors and hematologic malignancies. ${ }^{1}$ However, its clinical use is limited by neurotoxicity which can occur at any stage during or following treatment. $^{2}$ The anti-tumor activity of Dox has been reported to be mediated through the inhibition of the nuclear enzyme DNA topoisomerase II, causing DNA breakage, and generation of superoxide, hydrogen peroxide, and hydroxyl radicals. It is assumed that oxidative stress and free radical formation play a crucial role in the mechanism of Dox-induced organ toxicity.,4 Therefore, the use of the Dox is prone to cause tissue damage and adverse effects such as heart arrhythmias, neutropenia, cardiotoxicity, and neurotoxicity. Accumulating evidence showed that Dox decreases hippocampal neurogenesis and enhances inflammation, the result of which is always tightly related to the pathophysiological mechanisms of depression. ${ }^{5,6}$ Our previous 
study also demonstrated that prolonged use of Dox caused neural apoptosis and successfully induced depression-like behaviors in rats. ${ }^{7}$ However, the underlying mechanisms of Dox-induced neurotoxicity remain elusive.

NRG1, a protein encoded by the NRG1 gene, has been identified as an active EGF family member. ${ }^{8}$ NRG1 is a ligand for the ErbB family of tyrosine kinase transmembrane receptors, including the ErbB3 and ErbB4. ${ }^{9}$ After ligand binding, the ErbB receptors preferentially dimerize with ErbB2, which then activate the Akt and extracellular-regulated kinase (ERK) signaling pathway and trigger a diverse set of biological processes, including myelination, neurite outgrowth, cell proliferation, differentiation, and protection against apoptosis. ${ }^{10-12}$ NRG1 is suggested as a susceptibility gene for several psychiatric disorders, including schizophrenia, bipolar disorder, and depression. ${ }^{10,11} \mathrm{NRG1}$ is well known to play an essential role in neuronal development as well as in maintaining normal function in the mature nervous system. ${ }^{13}$ Recent biochemical studies have shown that NRG1 can be neuroprotective for cortical neurons, motor neurons, dopaminergic neurons, cochlear sensory neurons, and PC12 cells. ${ }^{14-18}$ Previous research showed that aberrant changes in these NRG1-related pathways are tightly linked to the pathogenesis of depression. ${ }^{19}$

It is well known that the NRG1/ErbB signal system plays a critical role in the development of the cardiovascular system and the maintenance of adult heart function. ${ }^{20}$ Interestingly, the NRG1/ErbB system is also involved in the Dox-induced cardiotoxicity. It has been reported that Dox treatment pronouncedly inhibits NRG1 expression and suppresses ErbB4 activation, whereas NRG1 treatment alleviates Dox-induced myocardial apoptosis and improves cardiac function. ${ }^{21,22}$ In addition, it has been reported that in the heterozygous knockout of NRG1 in mice, Dox induces more severe cardiac injury, higher mortality, and worse left ventricular dysfunction compared to wild-type mice. ${ }^{23}$ Given the critical role of the NRG1/ErbB system in both brain and heart, it is tempting to hypothesize that Dox may also inhibit neural NRG1/ErbB signaling, thereby triggering the neurotoxicity and behavioral changes. Therefore, in this study, we aimed to investigate the changes in the NRG1/ErbB signal system in the nervous system induced by Dox, to further clarify the potential relationship between Dox-induced neurotoxicity and NRG1/ErbB pathway.

\section{Materials and methods}

\section{Animals}

Male, Sprague-Dawley rats (200-230 g) supplied by the Experimental Animal Center of the Second Xiangya Hospital were housed under standard conditions of temperature $\left(23^{\circ} \mathrm{C} \pm 2^{\circ} \mathrm{C}\right)$ and light $(12: 12 \mathrm{~h}$ light/dark cycle), with free access to food and water, prior to sucrose preference test (SPT). All animal use procedures were carried out in accordance with the Regulations of Experimental Animal Administration issued by the State Committee of Science and Technology of the People's Republic of China, with the approval of the ethics committee in Central South University (protocol number 021/2016).

\section{Experimental design}

The animals were randomly divided into three groups of eight rats each ( $\mathrm{n}=8)$ : 1) control, 2) DoxS, and 3) DoxL. The untreated control group was injected with the appropriate volume of the normal saline. Rats in DoxS were given a total of three intraperitoneal injections of Dox (Zhejiang Hisun Pharmaceutical Company Limited, Taizhou, China) every 2 days at a dose of $2.5 \mathrm{mg} / \mathrm{kg}$, and the DoxL group was given a total of seven injections of Dox every 2 days in the same way. The body weight of these rats was monitored throughout the experiment, and the doses were adjusted to each rat's body weight change.

At the end of the 2 weeks, behavioral tests were carried out, and the rats were sacrificed under anesthesia. Blood was collected, and the brain was quickly removed after cardiac perfusion with phosphate-buffered saline (PBS) $(\mathrm{pH}=7.2)$. The left hemisphere of the brain was maintained in $4 \%$ paraformaldehyde and then embedded in paraffin and prepared for histopathological examination and immunohistochemical staining. For the right hemisphere, the hippocampus was dissected and used for Western blot and polymerase chain reaction $(\mathrm{PCR})$ analyses.

\section{Behavioral test \\ SPT}

SPT is widely used for the measurement of stress-induced anhedonia state, a key depressive-like behavior in rats. ${ }^{24}$ Prior to SPT, all the rats were housed individually and habituated to $48 \mathrm{~h}$ of forced $1 \%$ sucrose solution consumption in two bottles on each side. After 14-h water deprivation, we placed two pre-weighed bottles, one containing $1 \%$ sucrose solution and another containing tap water near to each rat. The side (left and right) of the two bottles was randomly placed in order to avoid spatial bias. The bottles were weighed again after $1 \mathrm{~h}$, and the weight difference was considered to be the rat intake from each bottle. The preference for sucrose was measured as a percentage of the consumed $1 \%$ sucrose solution relative to the total amount of liquid intake. 


\section{Forced swimming test (FST)}

FST is widely employed to screen antidepressant efficacy and depressive-like behavior in rodents. ${ }^{25}$ In brief, each rat was placed in a Plexiglas cylinder ( $45 \mathrm{~cm}$ height, $25 \mathrm{~cm}$ diameter) containing $\sim 35 \mathrm{~cm}$ of water $\left(24^{\circ} \mathrm{C} \pm 1^{\circ} \mathrm{C}\right)$ for $15 \mathrm{~min}$. The rats were then dried and placed in their home cage. Twenty-four hours later, the rats were exposed to the same experimental conditions outlined earlier for a 5-min FST. All of the procedures were conducted at 9 am to $3 \mathrm{pm}$. Each test session was videotaped, and the duration of immobility was scored by an experienced observer blind to the experiment design. Immobility was defined as floating passively and only making slight movements to keep the head above water. The water in the tank was changed after each test.

\section{Novelty-suppressed feeding test (NSFT)}

The NSFT was adapted from previous study ${ }^{26}$ Before testing, rats were food deprived for $24 \mathrm{~h}$ in their home cages. The rats were placed in an open field $(75 \times 75 \times 40 \mathrm{~cm})$ with a small amount of food placed on a piece of white paper $(10 \times 10 \mathrm{~cm})$ in the center. Animals were allowed to explore the open field for $8 \mathrm{~min}$. The latency to feed, specifically the time it took for the rat to approach and take the first bite of the food, was recorded by a stopwatch. Immediately afterwards, the animals were transferred to their home cage, and the total food intake for the next $5 \mathrm{~min}$ was also weighed to avoid the influence of the animals' appetite.

\section{Real-time PCR analysis}

Total RNA from the hippocampus was extracted using the Trizol (Thermo Fisher Scientific, Waltham, MA, USA) method as recommended by the manufacturer. We performed real-time PCRs of the gene expression of Bax, Bcl-xl, NRG1, ErbB4, and ErbB2. The quantity and integrity of RNA concentrations were determined using the spectrophotometry (Jingke, Ningbo, China). cDNA was produced using the Revert Aid First Strand cDNA Synthesis Kit (Thermo Fisher Scientific). Quantitative PCR was performed on the Bio-rad Cx96 Detection System (Bio-Rad Laboratories Inc,
Hercules, CA, USA) using SYBR green PCR kit (Thermo Fisher Scientific) and gene-specific primers. A 5 ng cDNA sample was used with 40 cycles of amplification. Each cDNA was tested in triplicate. Relative quantitation for the PCR product was normalized to $\beta$-actin as an internal standard. The sequences of gene-specific primers are listed in Table 1.

\section{Western blot analysis}

One day after the behavioral tests, rats were anesthetized with $10 \%$ chloral hydrate $(4 \mathrm{~mL} / \mathrm{kg})$, and the tissues were rapidly collected. For Western blot analysis, total protein was prepared from the hippocampus, and the concentration was determined using the Bradford method. Samples were loaded on a precast $12 \%$ sodium dodecyl sulfate polyacrylamide gel electrophoresis with $10 \mu \mathrm{g}$ protein in each lane. Proteins in the gels were transferred to a polyvinylidene fluoride membrane and blocked for $1 \mathrm{~h}$ in 5\% non-fat dry milk in TBS-T (25 mM Tris, pH 7.5, $150 \mathrm{mM} \mathrm{NaCl}, 0.05 \%$ Tween-20). The following antibodies and concentrations were used over night at $4{ }^{\circ} \mathrm{C}$; NRG1 (Santa Cruz Biotechnology Inc., Dallas, TX, USA, sc-28916; 1:400), ErbB4 (Santa Cruz Biotechnology Inc., sc-283; 1:500), ErbB2 (Cell Signaling, Danvers, MA, USA, 2165; 1:1,500), p-ErbB4 (Tyr1056) (Santa Cruz Biotechnology Inc., sc33040; 1:300), p-ErbB2 (Tyr1248) (Cell Signaling, 2247; 1:1,500), p-Akt (Ser473) (Cell Signaling, 4060; 1:3,000), p-ERK (Thr202/Tyr204) (Cell Signaling, 4695; 1:2,000), caspase-3 (Abcam, Cambridge, UK, ab4051; 1:500), and $\beta$-actin (Proteintech, Rosemont, IL, USA, 660091-Ig; 1:4,000). Membranes were then probed with horseradish peroxidase-conjugated secondary antibody for $40 \mathrm{~min}$. After washing, membranes were dipped in electrochemiluminescence, and immunoblots were analyzed by using the Bioprofil Biolight PC software (Vulber Lourmat, France). The signals were normalized to $\beta$-actin as an internal standard.

\section{Histopathological examination}

For histological tissue analysis, the tissues were fixed in $10 \%$ formalin, processed, and then were dehydrated in increasing concentrations of ethanol $(70 \%-100 \%)$ and embedded

Table I Primer sequences used for the qPCR analysis

\begin{tabular}{llll}
\hline Gene & Sense primer $\left(\mathbf{5}^{\prime} \mathbf{-} \mathbf{3}^{\prime}\right)$ & Antisense primer $\left(\mathbf{5}^{\prime} \mathbf{-} \mathbf{3}^{\prime} \mathbf{)}\right.$ & $\begin{array}{c}\text { Amplicon } \\
\text { length (bp) }\end{array}$ \\
\hline Bax & CCAGGACGCATCCACCAAGAAGC & TGCCACACGGAAGAAGACCTCTCG & I35 \\
Bcl-xl & CAGCTTCATATAACCCCAGGGAC & GCTCTAGGTGGTCATTCAGGTAGG & 207 \\
NRGI & GAGTCAGTTCAAGAGCCCGTTAA & GCCATTGGGCTTGGTTCTTT & 69 \\
ErbB4 & TGATTGCAGCCGGAGTCAT & TGACATAAACGGCAAATGTCAGA & 181 \\
ErbB2 & CCTGCCTCCACTTCAATCAT & CAGGATCCCACTTCCGTAGA & 172 \\
$\beta$-actin & CATCCTGCGTCTGGACCTGG & TAATGTCACGCACGATTTCC & 116 \\
\hline
\end{tabular}

Abbreviation: qPCR, quantitative polymerase chain reaction. 
in paraffin. The paraffin tissue blocks were prepared for sectioning at $5 \mu \mathrm{m}$ thickness using a sledge microtome. Paraffin sections were stained with hematoxylin and eosin (H\&E) for light microscopic examination. The histology was assessed by a pathologist who was blind to the treatment groups.

\section{Immunohistochemical study}

Paraffin-embedded tissue sections of $5 \mu \mathrm{m}$ thickness were rehydrated first in xylene and then in graded ethanol solutions. The slides were then blocked with $5 \%$ bovine serum albumin (BSA) in tris-buffered saline (TBS) for $2 \mathrm{~h}$. The sections were then immunostained with the primary monoclonal to rat caspase-3 antibody (Abcam, ab4051; 1:100) at a concentration of $1 \mathrm{mg} / \mathrm{mL}$ containing 5\% BSA in TBS and incubated overnight at $4^{\circ} \mathrm{C}$. After washing the slides with TBS, the sections were incubated with goat anti-rabbit secondary antibody. Sections were then washed with TBS and incubated for 5-10 min in a solution of $0.02 \%$ diaminobenzidine containing $0.01 \%$ $\mathrm{H}_{2} \mathrm{O}_{2}$. Counter staining was performed using hematoxylin, and the slides were visualized under a light microscope.

\section{Statistical analysis}

All statistical procedures were performed using SPSS version 13.0 software (SPSS Inc., Chicago, IL, USA). Data were expressed as mean \pm standard error of the mean (SEM) and analyzed statistically by one-way analysis of variance (ANOVA) with Dunnett's $t$-test for post hoc multiple comparisons. The prior level of significance was established at $p<0.05$.

\section{Results}

\section{Effects of Dox on body weight gain and behavioral changes}

The body weight of rats in the three groups was basically the same before experiment (Figure 1A). The Dox-treated rats showed significantly decreased body weight when compared to control animals (Figure $1 \mathrm{~B}, p<0.01$ ), which is consistent with the results of the previous study. ${ }^{27}$ In the experiment of SPT, the DoxL group exhibited a significant decrease in the sucrose preference (Figure $1 \mathrm{C}, p<0.05$ ) when compared to the other two groups. The immobility time was recorded in the FST, and we found that administration of Dox for seven times led to a significant enhancement in the immobility time (Figure $1 \mathrm{D}, p<0.01$ ) when compared to the other two groups. The latency time and food intake were observed in the NSFT, the latency time was significantly increased in both DoxS and DoxL groups (Figure 1E, $p<0.01$ ), and there was no significant difference of food intake in the three groups (Figure 1F).

\section{Effects of Dox on neural apoptosis}

Histopathological alterations in hippocampus specimens from different treated groups are shown in Figure 2A. The hippocampus tissues from the control group showed normal histology, while Dox-administered groups showed more frequent nuclear pyknosis, and the phenomenon was more obvious in the DoxL group. The expression of caspase-3 was estimated using immunohistochemical staining (Figure 2B). The control group showed minimal immunoreaction for caspase-3, while the administration of Dox induced the expression of the marker by the intense brown staining as compared to the control group, and the administration of Dox for seven times increased the expression of caspase-3. Similarly, the result of the Western blot analysis of caspase- 3 in the hippocampus tissues was in accordance with the immunohistochemical staining. The expression of caspase-3 was significantly enhanced in both DoxS and DoxL groups (Figure $2 \mathrm{C}, p<0.01$ ). Consistent with the results of Western blot analysis of caspase-3, a significant increase in gene expression of pro-apoptotic Bax was observed after administration of Dox (Figure 2D, $p<0.01$ ). The gene expression of anti-apoptotic factors (Bcl-xl) was increased after the administration of Dox for three times (Figure 2E, $p<0.05$ ).

\section{Effects of Dox on brain NRG I/ErbB expression}

As shown in Figure 3A, administration of Dox for three times significantly increased the gene expression of NRG1 $(p<0.01)$, whereas gene expression of NRG1 was not influenced in rats treated with Dox for seven times in our study. Consistent with the gene expression, the expression of NRG1 was significantly enhanced in the DoxS group (Figure 3B, $p<0.01$ ), while the expression of NRG1 was decreased in the DoxL group (Figure 3B, $p<0.05$ ). As shown in Figure 4, although gene expression of ErbB4 and ErbB2 in the hippocampus was not statistically changed in our study, the ratios of pErbB4/ErbB4 (Figure 4B, $p<0.01$ ) and $\mathrm{pErbB} 2 / \mathrm{ErbB} 2$ (Figure 4D, $p<0.01$ ) were significantly decreased after administration of Dox for seven times. The ratios of $\mathrm{pErbB} 4 / \mathrm{ErbB} 4$ (Figure 4B, $p<0.01$ ) and $\mathrm{pErbB} 2 /$ ErbB2 (Figure 4D, $p<0.05$ ) were significantly increased in the DoxS group.

\section{Effects of Dox on the activation of Akt and ERK in rats}

The activation of Akt and ERK by Dox is shown in Figure 5. The ratios of $\mathrm{pAkt} / \mathrm{Akt}$ (Figure $5 \mathrm{~A}, p<0.01$ ) and $\mathrm{pERK} / \mathrm{ERK}$ (Figure $5 \mathrm{~B}, p<0.01$ ) were significantly decreased in the 
A

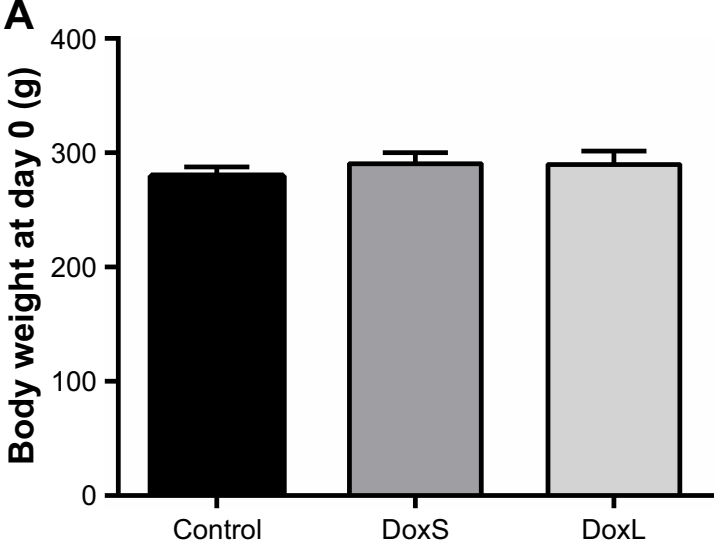

C

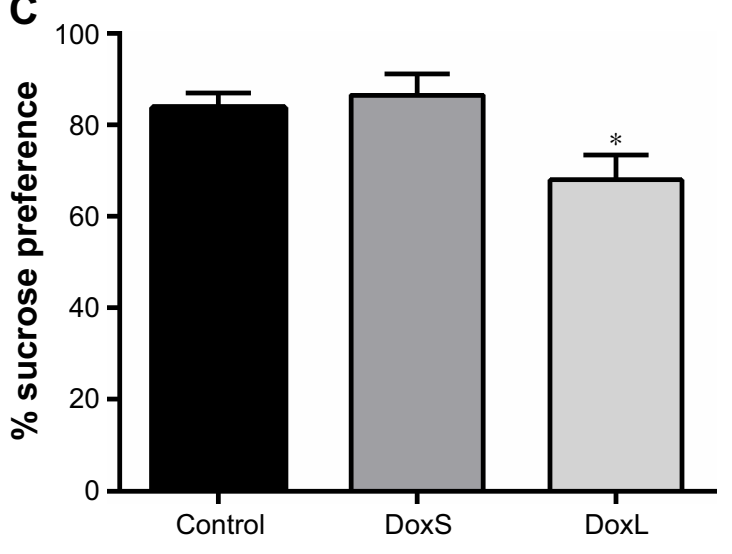

E

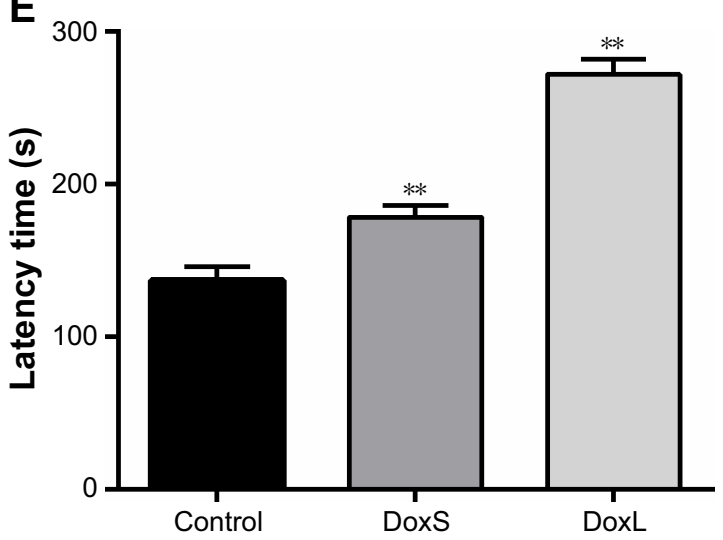

B

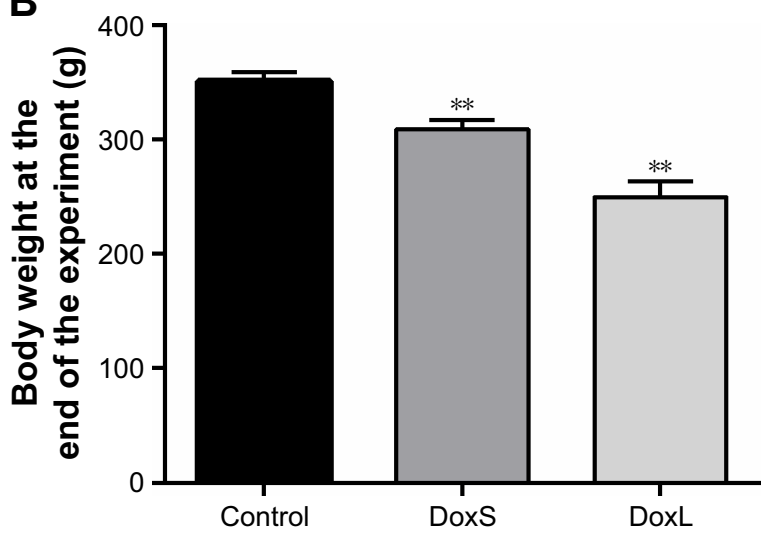

D

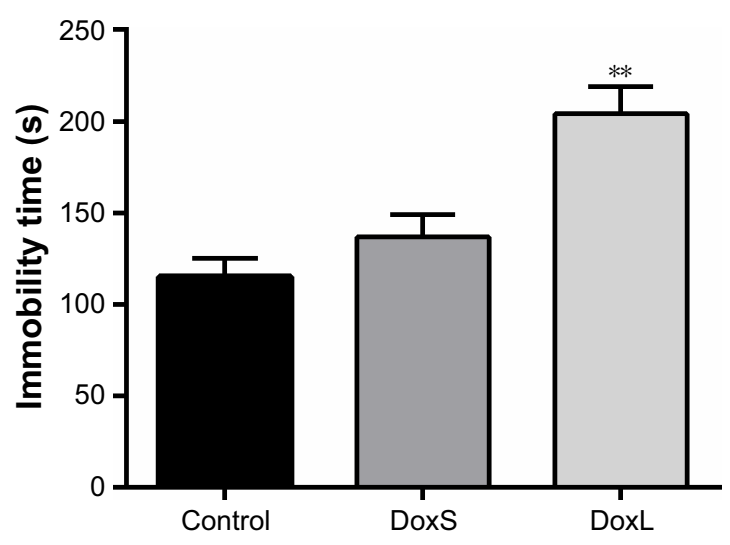

$\mathbf{F}$

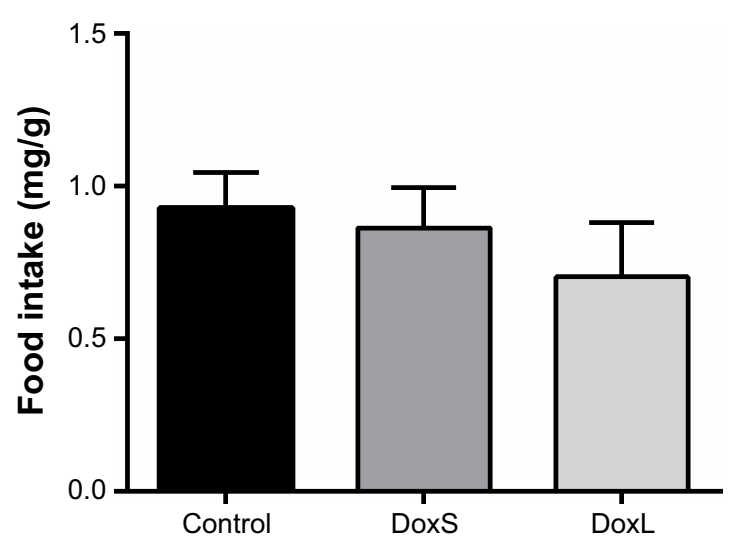

Figure I Effects of Dox on body weight gain and behavioral changes.

Notes: Effect of Dox on body weight gain (A, B); effect of Dox on sucrose preference test and forced swimming test: sucrose preference (C); immobility time (D); effect of Dox on novelty suppressed feeding test: latency time $(\mathbf{E})$; and food intake $(\mathbf{F})$. Data are mean \pm SEM ( $n=6-7)$. ${ }^{*} p<0.05$ and $* * p<0.01$ compared to the related control group. Abbreviations: Dox, doxorubicin; DoxS, doxorubicin administration for short time; DoxL, doxorubicin administration for long time; SEM, standard error of the mean.

DoxL group, whereas the phosphorylation level of Akt was significantly enhanced after administration of Dox for three times (Figure $5 \mathrm{~A}, p<0.01$ ).

\section{Discussion}

NRG1 is expressed at synaptic regions in many brain areas, which is involved in many key neuronal developmental functions, including neuronal migration, synaptic modulation, and oligodendrocyte development. NRG1 has been reported to prevent brain injury following stroke and to exert a protective role for dopaminergic neurons in a mouse model of Parkinson's disease. ${ }^{28} \mathrm{~A}$ burgeoning body of evidence suggests that NRG1 is associated with traumatic brain injury and Alzheimer's disease. ${ }^{29}$ 
A
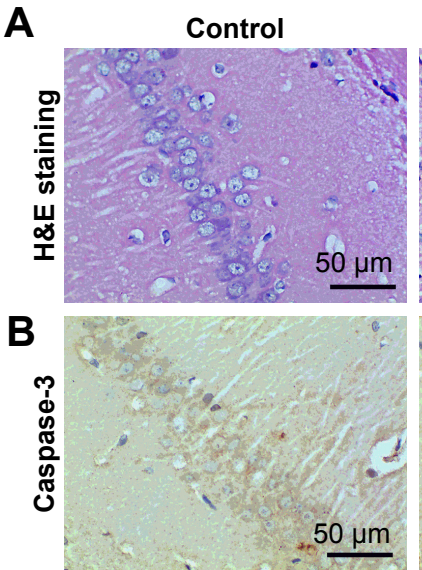
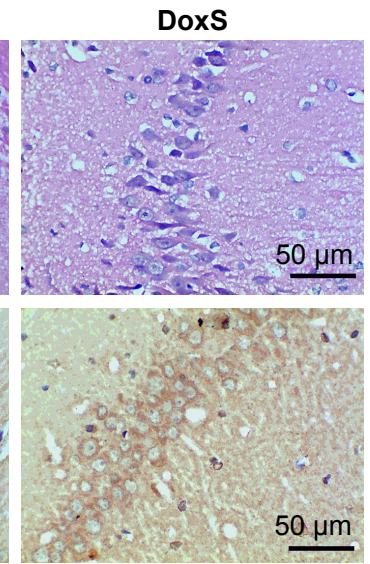
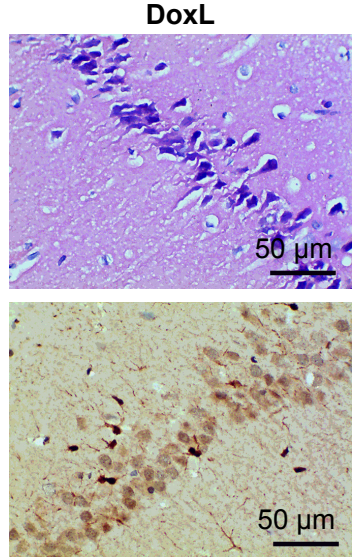

\section{C

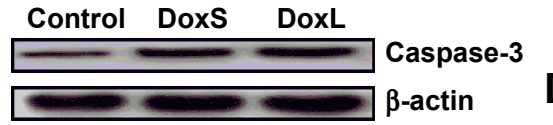

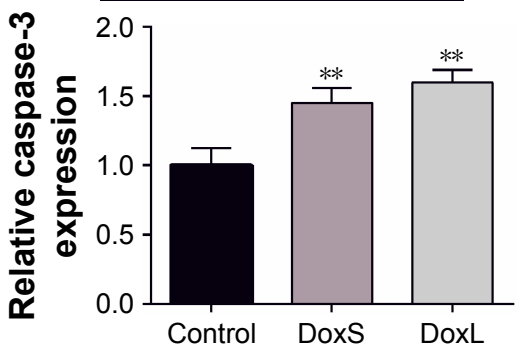

D

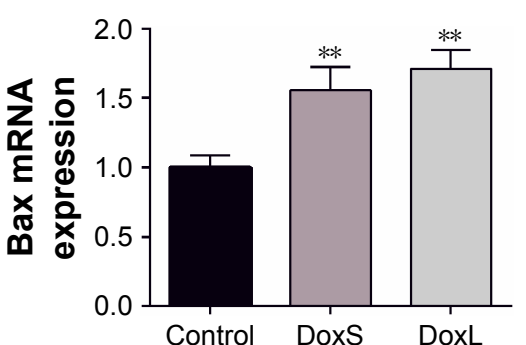

E

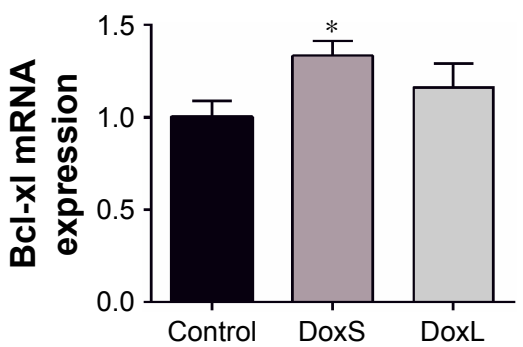

Figure 2 Effects of Dox on neural apoptosis.

Notes: H\&E staining in the hippocampus specimens (A); immunohistochemical staining of caspase-3 (B); Western blot analysis of caspase-3 (C); gene expression of pro-apoptotic factors (Bax) (D) and anti-apoptotic factors (Bcl-xl) (E). Data are expressed as mean \pm SEM ( $n=6-7)$. $* p<0.05$ and $* * p<0.01$ compared to control group. Magnification: $\times 400$.

Abbreviations: Dox, doxorubicin; DoxS, doxorubicin administration for short time; DoxL, doxorubicin administration for long time; H\&E, hematoxylin and eosin; Bax, Bcl2-associated X; Bcl2, B cell leukemia/lymphoma 2; SEM, standard error of the mean.

Several genetic linkage analysis and genome-wide association studies identified NRG1 as a susceptibility gene of major depressive disorder, which was validated by its role in neurodevelopment, glutamate, and other neurotransmitter receptor expression regulation. ${ }^{30}$ Evidence showed that aberrant changes in NRG1-related pathways are tightly linked to the pathogenesis of depression, and the administration of NRG1 could improve the nerve function in patients with neurological disorders, indicating a promising therapeutic target for depression. ${ }^{19}$ Wen et a ${ }^{30}$ also reported that NRG1 is

\section{A}

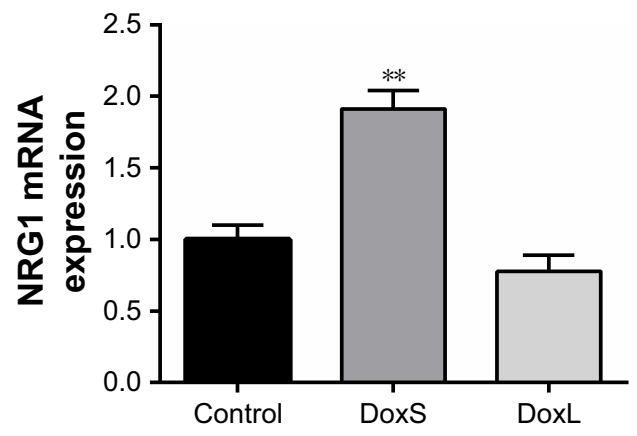

B

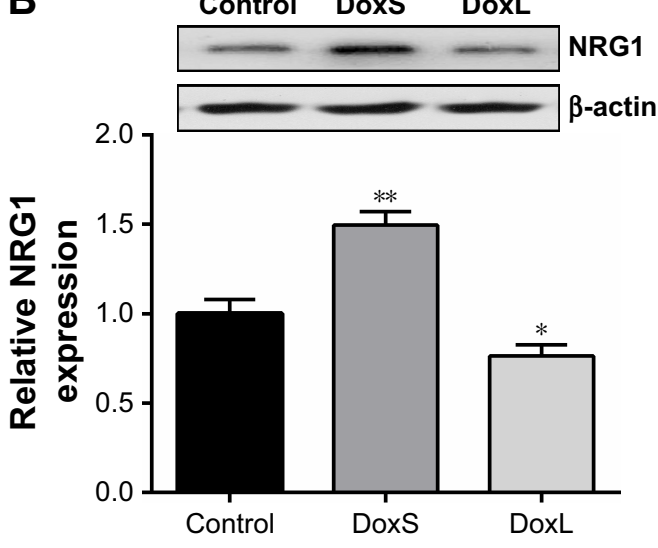

Figure 3 Effect of Dox on gene expression and relative expression of NRGI.

Notes: NRGI mRNA expression (A) and relative NRGI expression (B). Data are expressed as mean $\pm S E M(n=6-7)$. ${ }^{*} p<0.05$ and $* * p<0.01$ compared to the control group. Abbreviations: Dox, doxorubicin; DoxS, doxorubicin administration for short time; DoxL, doxorubicin administration for long time; NRGI, Neuregulin I; SEM, standard error of the mean. 


\section{A}

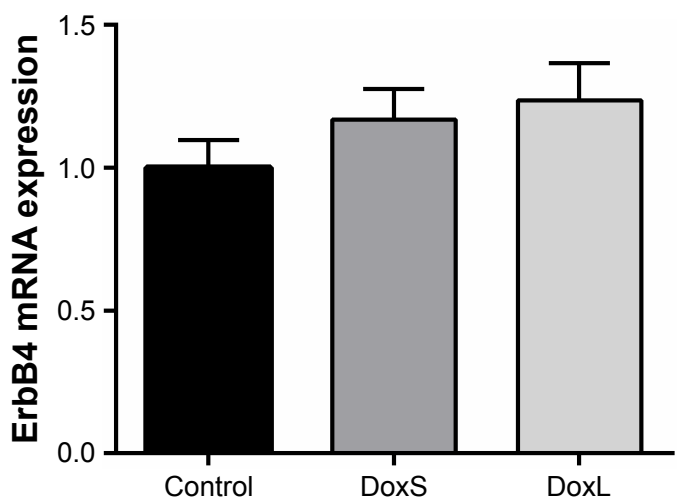

C

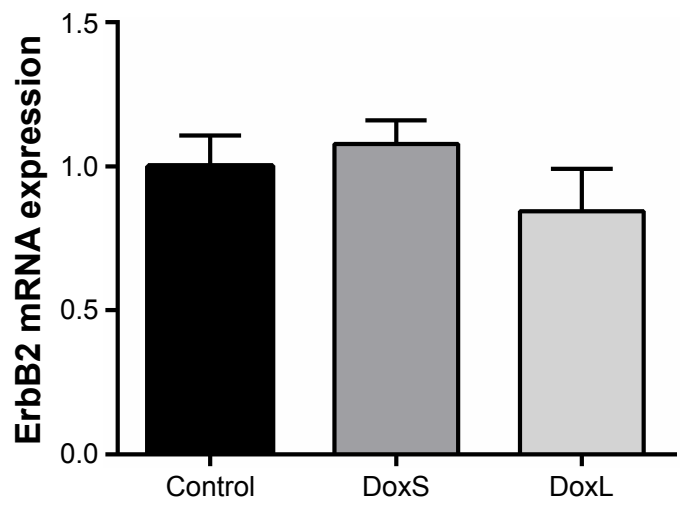

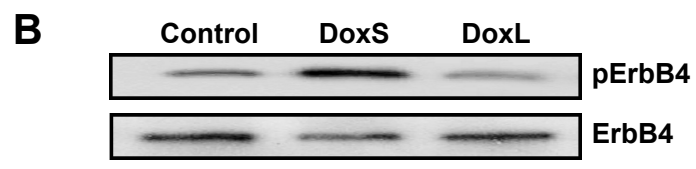
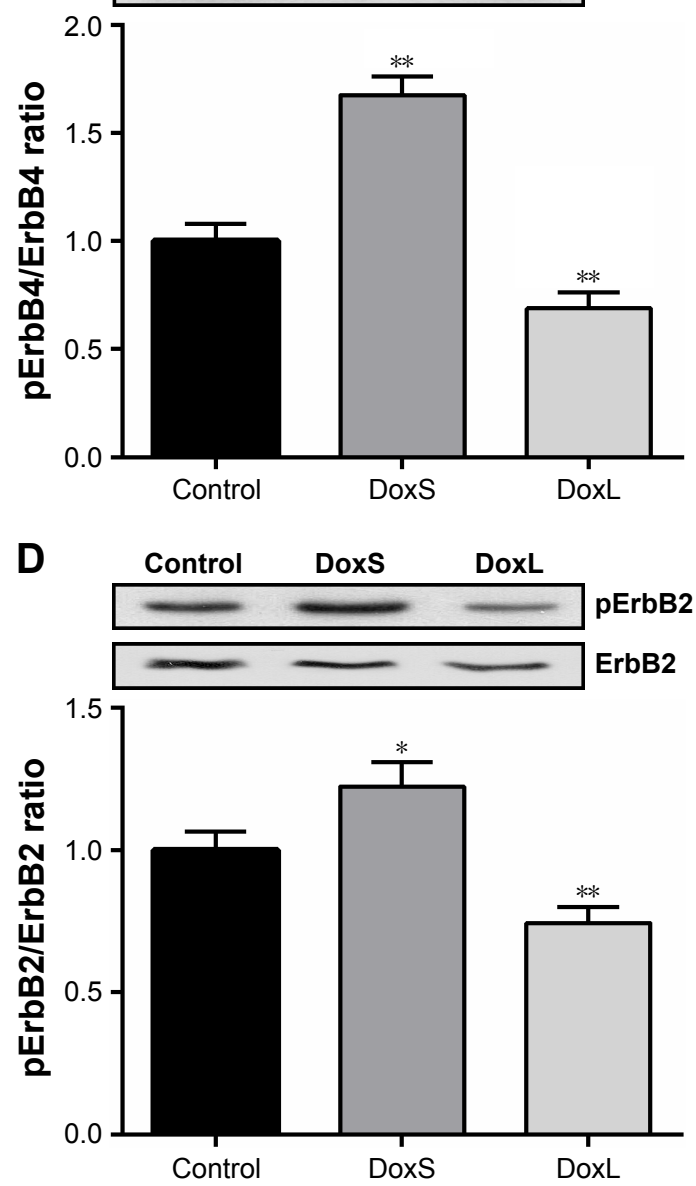

Figure 4 Effect of Dox on gene expression of ErbB2, ErbB4, and the ratio of pErbB4/ErbB4 and pErbB2/ErbB2 in the hippocampus.

Notes: ErbB4 mRNA expression (A); pErbB4/ErbB4 ratio (B); ErbB2 mRNA expression (C); and pErbB2/ErbB2 ratio (D). Data are expressed as mean \pm SEM ( $\mathrm{n}=6-7$ ). $* p<0.05$ and $*^{*} p<0.0$ l compared to the control group.

Abbreviations: Dox, doxorubicin; DoxS, doxorubicin administration for short time; DoxL, doxorubicin administration for long time; SEM, standard error of the mean.

A
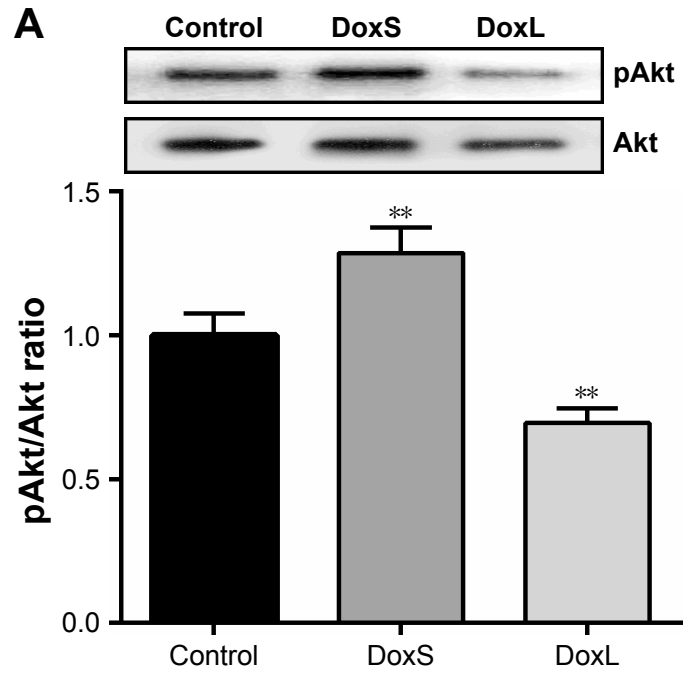

B
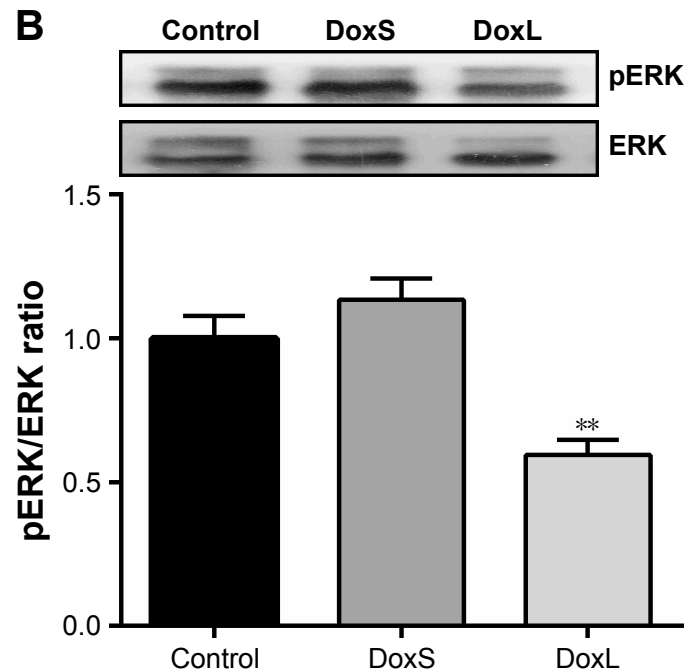

Figure 5 Effects of Dox on the activation of Akt and ERK.

Notes: pAkt/Akt ratio (A) and pERK/ERK ratio (B). Data are expressed as mean \pm SEM $(n=6-7)$. ${ }^{* *} p<0.01$ compared to the control group

Abbreviations: Dox, doxorubicin; DoxS, doxorubicin administration for short time; DoxL, doxorubicin administration for long time; SEM, standard error of the mean. 
a common susceptibility gene for major depressive disorder in Han population. Similarly, Bi et $\mathrm{al}^{31}$ also found that the endogenous NRG1-ErbB4 signaling pathway in the basolateral amygdala is critical for the stress-induced behavioral changes, including major depression, and the administration of NRG1 into the basolateral amygdala of high-anxiety mice alleviates their anxiety and enhances GABAergic neurotransmission.

Dox-induced cardiotoxicity and neurotoxicity are dose-related and essentially irreversible, and the long-term use of Dox tends to induce neurotoxicity and may cause neuropsychiatric diseases including depression. ${ }^{3}$ In vivo study has shown that Dox-induced cardiovascular disease is tightly associated with the suppression of NRG1/ErbB signaling, whereas the activation of NRG1/ErbB signaling promotes cardiac protection during acute cardiac injury and chronic ventricular remodeling. ${ }^{21}$ However, the potential link between Dox-induced neurotoxicity and NRG1/ErbB pathway remains largely unknown. To fill this critical gap, the present study first evaluated the suppression of the NRG1/ ErbB pathway in the brain after administration of Dox.

The results of our study showed that the administration of Dox for three times activated the NRG1/ErbB pathway, but the pathway was suppressed after administration of Dox for a long time (seven times) and induced a series of depressionlike behaviors. The administration of Dox for three times induced higher expression of NRG1 and also promoted the phosphorylation level of Akt and ERK, which indicated the adaptive response to the acute brain damage. Consistent with our study, Salas-Ramirez et al also found that the rats treated for three times with a combination of cyclophosphamide and Dox show significantly activated ERK and Akt signaling pathways. ${ }^{32}$ The results of the previous behavioral testing also demonstrated that chemotherapy can impair cognitive function, without affecting anxiety. ${ }^{32}$ Interestingly, our studies found that NRG1/ErbB signaling and the downstream Akt and ERK pathway were significantly suppressed in the DoxL group, resulting in neurotoxicity and behavioral changes. This biphasic response of the NRG1/ErbB signaling pathway has also been found in heart. The NRG1 is adaptively activated in the prophase of heart disease and is also suppressed with the development of cardiovascular disease. Consistent with our report, Horie et al have found that the acute cardiotoxicity induced by Dox is associated with the inhibition of the NRG1-ErbB pathway, ${ }^{33}$ and studies also showed that the supplementation of NRG1 is effective in attenuating Dox-induced cardiac dysfunction in mouse hearts. Thus, as a potential therapeutic agent for the treatment of heart failure and nerve damage, the NRG1/ErbB signaling pathway might

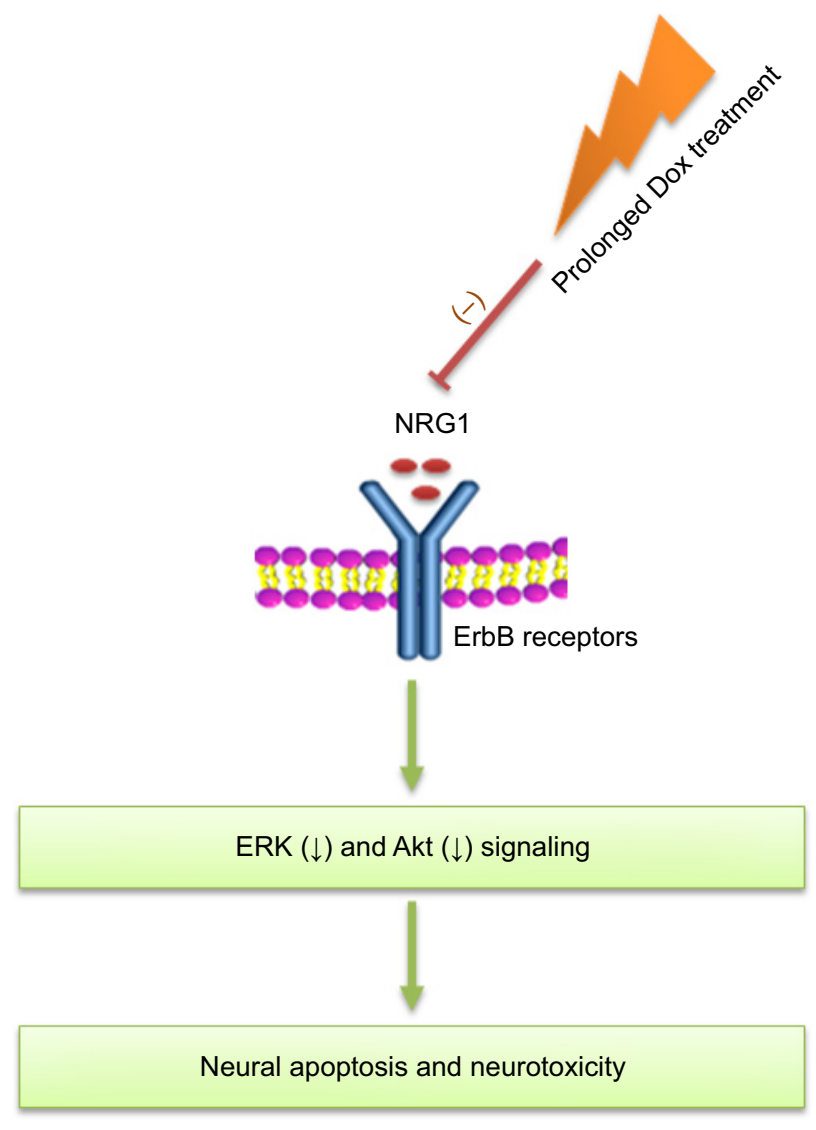

Figure 6 Proposed mechanisms of the Dox-induced neurotoxicity. Note: The drug-induced dysregulation of Neuregulin-I/ErbB pathway further suppresses downstream ERK and Akt signaling and results in the behavioral changes. Abbreviations: Dox, doxorubicin; ERK, extracellular-regulated kinase; NRGI, Neuregulin-I.

be the underlying mechanisms of Dox-induced tissue damage in both heart and brain (Figure 6).

\section{Conclusion}

The present study showed for the first time that the administration of Dox could lead to the alteration of the NRG1/ ErbB signaling pathway and dysregulation of the downstream Akt and ERK pathway in rats, which raised an intriguing possibility for the involvement of the NRG1/ErbB pathway in Dox-induced neurotoxicity. Although the underlying mechanism should be further explored, our study provided new evidence for the research of the role of the NRG1/ErbB signaling pathway in Dox-induced neurotoxicity and a potential target for the Dox-induced comorbidity of depression and cardiotoxicity.

\section{Acknowledgment}

This study was supported by National Natural Science Foundation of China (NSFC: 81603206) and Natural Science Foundation of Hunan province (NO: 2015JJ6062). 


\section{Disclosure}

The authors report no conflicts of interest in this work.

\section{References}

1. Eisa NH, ElSherbiny NM, Shebl AM, Eissa LA, El-Shishtawy MM. Phenethyl isothiocyanate potentiates anti-tumour effect of doxorubicin through Akt-dependent pathway. Cell Biochem Funct. 2015;33(8): 541-551.

2. Xiang P, Deng HY, Li K, et al. Dexrazoxane protects against doxorubicin-induced cardiomyopathy: upregulation of Akt and Erk phosphorylation in a rat model. Cancer Chemother Pharmacol. 2009; 63(2):343-349.

3. Rocha P, Campos JF, Nunes-Souza V, et al. Antioxidant and protective effects of schinus terebinthifolius raddi against doxorubicin-induced toxicity. Appl Biochem Biotechnol. Epub 2017 Sep 9.

4. Cheruku SP, Ramalingayya GV, Chamallamudi MR, et al. Catechin ameliorates doxorubicin-induced neuronal cytotoxicity in in vitro and episodic memory deficit in in vivo in Wistar rats. Cytotechnology. Epub 2017 Sep 13.

5. Seigers R, Fardell JE. Neurobiological basis of chemotherapy-induced cognitive impairment: a review of rodent research. Neurosci Biobehav Rev. 2011;35(3):729-741.

6. Wigmore P. The effect of systemic chemotherapy on neurogenesis, plasticity and memory. Curr Top Behav Neurosci. 2013;15:211-240.

7. Wu YQ, Dang RL, Tang MM, et al. Long chain omega-3 polyunsaturated fatty acid supplementation alleviates doxorubicin-induced depressive-like behaviors and neurotoxicity in rats: involvement of oxidative stress and neuroinflammation. Nutrients. 2016;8(4):243.

8. Ritch PS, Carroll SL, Sontheimer H. Neuregulin-1 enhances survival of human astrocytic glioma cells. Glia. 2005;51(3):217-228.

9. Gui C, Zhu L, Hu M, Lei L, Long Q. Neuregulin-1/ErbB signaling is impaired in the rat model of diabetic cardiomyopathy. Cardiovasc Pathol. 2012;21(5):414-420.

10. Liu J, Kern JA. Neuregulin-1 activates the JAK-STAT pathway and regulates lung epithelial cell proliferation. Am J Respir Cell Mol Biol. 2002;27(3):306-313.

11. Puricelli L, Proietti CJ, Labriola L, et al. Heregulin inhibits proliferation via ERKs and phosphatidyl-inositol 3-kinase activation but regulates urokinase plasminogen activator independently of these pathways in metastatic mammary tumor cells. Int $J$ Cancer. 2002;100(6): 642-653.

12. Kamezaki A, Sato F, Aoki K, et al. Visualization of Neuregulin 1 ectodomain shedding reveals its local processing in vitro and in vivo. Sci Rep. 2016;6:28873.

13. Corfas G, Roy K, Buxbaum JD. Neuregulin 1-erbB signaling and the molecular/cellular basis of schizophrenia. Nat Neurosci. 2004; 7(6):575-580.

14. Goldshmit Y, Erlich S, Pinkas-Kramarski R. Neuregulin rescues PC12-ErbB4 cells from cell death induced by $\mathrm{H}(2) \mathrm{O}(2)$. Regulation of reactive oxygen species levels by phosphatidylinositol 3-kinase. J Biol Chem. 2001;276(49):46379-46385.

15. Li BS, Ma W, Jaffe H, et al. Cyclin-dependent kinase-5 is involved in neuregulin-dependent activation of phosphatidylinositol 3-kinase and Akt activity mediating neuronal survival. J Biol Chem. 2003; 278(37):35702-35709.

Drug Design, Development and Therapy

\section{Publish your work in this journal}

Drug Design, Development and Therapy is an international, peerreviewed open-access journal that spans the spectrum of drug design and development through to clinical applications. Clinical outcomes, patient safety, and programs for the development and effective, safe, and sustained use of medicines are the features of the journal, which
16. Ricart KJ, Pearson RJ, Viera L, et al. Interactions between betaneuregulin and neurotrophins in motor neuron apoptosis. JNeurochem. 2006;97(1):222-233.

17. Stankovic K, Rio C, Xia A, et al. Survival of adult spiral ganglion neurons requires erbB receptor signaling in the inner ear. $J$ Neurosci. 2004;24(40):8651-8661.

18. Zhang L, Fletcher-Turner A, Marchionni MA, et al. Neurotrophic and neuroprotective effects of the neuregulin glial growth factor- 2 on dopaminergic neurons in rat primary midbrain cultures. $J$ Neurochem. 2004;91(6):1358-1368.

19. Dang R, Cai H, Zhang L, et al. Dysregulation of Neuregulin-1/ErbB signaling in the prefrontal cortex and hippocampus of rats exposed to chronic unpredictable mild stress. Physiol Behav. 2016;154:145-150.

20. Dang R, Guo Y, Zhang L, Chen L, Yang R, Jiang P. Chronic stress and excessive glucocorticoid exposure both lead to altered Neuregulin-1/ ErbB signaling in rat myocardium. Steroids. 2016;112:47-53.

21. Vasti C, Hertig CM. Neuregulin-1/erbB activities with focus on the susceptibility of the heart to anthracyclines. World J Cardiol. 2014; 6(7):653-662.

22. Yan X, Morgan JP. Neuregulin1 as Novel Therapy for Heart Failure. Curr Pharm Des. 2011;17(18):1808-1817.

23. Liu FF, Stone JR, Schuldt AJ, et al. Heterozygous knockout of neuregulin-1 gene in mice exacerbates doxorubicin-induced heart failure. Am J Physiol Heart Circ Physiol. 2005;289(2):H660-H666.

24. Maniam J, Morris MJ. Long-term postpartum anxiety and depression-like behavior in mother rats subjected to maternal separation are ameliorated by palatable high fat diet. Behav Brain Res. 2010;208(1):72-79.

25. Rinwa P, Kumar A. Quercetin suppress microglial neuroinflammatory response and induce antidepressant-like effect in olfactory bulbectomized rats. Neuroscience. 2013;255:86-98.

26. Hamani C, Diwan M, Macedo CE, et al. Antidepressant-like effects of medial prefrontal cortex deep brain stimulation in rats. Biol Psychiatry. 2010;67(2):117-124.

27. El-Sayed E-SM, Mansour AM, El-Sawy WS. Protective effect of proanthocyanidins against doxorubicin-induced nephrotoxicity in rats. J Biochem Mol Toxicol. 2017;31(11):e21965.

28. Carlsson T, Schindler FR, Hollerhage M, et al. Systemic administration of neuregulin-1beta1 protects dopaminergic neurons in a mouse model of Parkinson's disease. J Neurochem. 2011;117(6):1066-1074.

29. Jiang Q, Chen S, Hu C, Huang P, Shen H, Zhao W. Neuregulin-1 (Nrg1) signaling has a preventive role and is altered in the frontal cortex under the pathological conditions of Alzheimer's disease. Mol Med Rep. 2016; 14(3):2614-2624.

30. Wen Z, Chen J, Khan RA, et al. Genetic association between NRG1 and schizophrenia, major depressive disorder, bipolar disorder in Han Chinese population. Am J Med Genet B Neuropsychiatr Genet. 2016; 171B(3):468-478.

31. Bi LL, Sun XD, Zhang J, et al. Amygdala NRG1-ErbB4 is critical for the modulation of anxiety-like behaviors. Neuropsychopharmacology. 2015;40(4):974-986.

32. Salas-Ramirez KY, Bagnall C, Frias L, Abdali SA, Ahles TA, Hubbard K. Doxorubicin and cyclophosphamide induce cognitive dysfunction and activate the ERK and AKT signaling pathways. Behav Brain Res. 2015;292:133-141.

33. Horie T, Ono K, Nishi H, et al. Acute doxorubicin cardiotoxicity is associated with miR-146a-induced inhibition of the neuregulin-ErbB pathway. Cardiovasc Res. 2010;87(4):656-664.

\section{Dovepress}

has also been accepted for indexing on PubMed Central. The manuscript management system is completely online and includes a very quick and fair peer-review system, which is all easy to use. Visit http://www.dovepress.com/testimonials.php to read real quotes from published authors. 